\title{
Article
}

\section{Eye-movements reveal attention to social information in autism spectrum disorder}

Fletcher-Watson, S., Leekam, S.R., Benson, Valerie, Frank, M.C. and Findlay, J.M.

Available at http://clok.uclan.ac.uk/28073/

Fletcher-Watson, S., Leekam, S.R., Benson, Valerie ORCID: 0000-0002-03514563, Frank, M.C. and Findlay, J.M. (2009) Eye-movements reveal attention to social information in autism spectrum disorder. Neuropsychologia, 47 (1). pp. 248-257. ISSN 0028-3932

It is advisable to refer to the publisher's version if you intend to cite from the work. http://dx.doi.org/10.1016/j.neuropsychologia.2008.07.016

For more information about UCLan's research in this area go to http://www.uclan.ac.uk/researchgroups/ and search for <name of research Group>.

For information about Research generally at UCLan please go to http://www.uclan.ac.uk/research/

All outputs in CLoK are protected by Intellectual Property Rights law, including Copyright law. Copyright, IPR and Moral Rights for the works on this site are retained by the individual authors and/or other copyright owners. Terms and conditions for use of this material are defined in the policies page. 

Elsevier Editorial System(tm) for Neuropsychologia

Manuscript Draft

Manuscript Number: NSY-D-08-00406R1

Title: Eye-movements reveal attention to social information in autism spectrum disorder

Article Type: Research Report

Section/Category: Emotion and Social Neuroscience

Keywords: eye-tracking; social attention; scene viewing; preferential-looking; perception.

Corresponding Author: Dr Sue Fletcher-Watson, Ph. D.

Corresponding Author's Institution: Newcastle University

First Author: Sue Fletcher-Watson, Ph. D.

Order of Authors: Sue Fletcher-Watson, Ph. D.; Susan R Leekam, PhD; Valerie Benson, PhD; Michael C Frank; John M Findlay, PhD

Abstract: Autism spectrum disorder (ASD) is a neurodevelopmental condition in which children show reduced attention to social aspects of the environment. However in adults with ASD, evidence for social attentional deficits is equivocal. One problem is that many paradigms present social information in an unrealistic, isolated way. This study presented adults and adolescents, with and without ASD, with a complex social scene alongside another, non-social scene, and measured eye-movements during a threesecond viewing period. Analyses first identified viewing time to different regions and then investigated some more complex issues. These were: the location of the very first fixation in a trial (indicating attentional priority); the effect of a task instruction on scan paths; the extent to which gaze-following was evident; and the degree to which participants' scan paths were influenced by the low-level properties of a scene. Results indicate a superficially normal attentional preference for social information in adults with ASD. However, more sensitive measures show that ASD does entail social attention problems across the lifespan, supporting accounts of the disorder which emphasise lifelong neurodevelopmental atypicalities. These subtle abnormalities may be sufficient to produce serious difficulties in real-life scenarios. 

Ref: NSY-D-08-00406

Dear Dr Tranel,

Please find attached a resubmission of our article entitled "Eye-movements reveal attention to social information in autism spectrum disorder". We are very grateful for the opportunity to respond to reviewers' comments and re-submit our work. Since Reviewer 1 had no revision suggestions, the new draft includes appropriate changes according Reviewer 2 only. I list here each major and minor comment and the action we have taken to address this concern.

\section{Reviewer 2: Major comments}

Comment: The authors pay little attention to the possible underlying mechanisms involved in their results pattern.

NB: this comment is also reiterated in the editor's letter

Action: The introduction now includes a more detailed description of the neural mechanisms thought to underpin social attention problems in autism, highlighted in grey on pages $3-4$. These issues are also reviewed in the Discussion on pages $23-24$

Comment: Please refer to Speer, Cook, McMahon \& Clark 2007.

Action: added on page 5, highlighted in grey

Comment: ... it would be worthwhile adding here that the trajectory of development might be impacted upon

Action: this is now addressed on page 4, paragraph 2, highlighted in grey

\section{Minor comments}

Comment: Page 2: change the misleading term 'social scenes'

Action: changed to "moving image with social content"

Comment: Page 3: add Speer at al

Action: added on page 5, highlighted in grey

Comment: Page 3: re-word paragraph beginning "Research measuring"

Action: as suggested

Comment: Page 3: make specific predictions

Action: We still feel that the existing evidence is so equivocal as to prevent concrete predictions being made but have more clearly delineated our reasoning on page 6 , highlighted in grey

Comment: Page 4: clarify final paragraph

Action: this has been re-written

Comment: Page 4: describe diagnostic information and/or exclusion criteria for TD participants

Action: this has been inserted, now on page 6 , highlighted in grey

Comment: Page 5: justify group-wise matching of participants (rather than one-to-one)

Action: as requested, now on page 7 , highlighted in grey 
Comment: Page 5: more detail is needed regarding stimuli - content categories, actor gender, actor location in the scene, facial expression,

Action: This information has now been added, on page 8, paragraph one, highlighted in grey. In particular, it should be noted that all actors had neutral facial expressions so no analysis of emotion processing or judgement was made.

Comment: Page 7: move IQ testing info to participant section

Action: $\quad$ this information has been moved to page 7, para 2.

Comment: Page 7: check typo in reference in Procedure section

Action: amended as requested

Comment: Page 8-10: emphasise group results throughout results section and make results more succinct

Action: Throughout, group effects (or the lack of them) have been moved to the start of the report of each new test. However, results have not been significantly cut. While we acknowledge that the results section is long, we feel this level of detail is required to clearly illustrate our findings and particularly to demonstrate how group differences are only apparent at the most subtle level of analysis. We would draw your attention to Reviewer 1's assertion that "the interim summaries are useful stepping stones which prepare the reader well for the final discussion", which we feel supports the existing presentation of our results section.

Comment: Page 11: acknowledge social-communicative importance of mouth region

Action: this issue is now addressed in the summary of viewing time results on page 1415 , highlighted in grey

Comment: Page 14: reference to Tables 5 and $6 ?$

Action: this has been amended - the reference is now to Table 4

Comment: Page 14: include time taken to make the first fixation in each domain of interest

Action: As is noted on page 16, highlighted in blue, there were no group differences in latency of first saccades made to any domain. Therefore, in the interests of preventing the results section becoming overcrowded we have decided not to include data on first saccade latency.

Comment: Page 14: typos

Action: amended as suggested

Comment: Page 14: more group-wise comparisons for PP and PA first fixations

Action: These have been added, highlighted in grey, on page 16

Comment: Page 16: clearer detail regarding analysis of looking where others look - defining the $30^{\circ}$ cone, link between eye and head direction, visibility of eye region, presence of target item in each scene

Action: The choice and application of the $30^{\circ}$ cone has been made clear, in text highlighted in grey on page 18.

We feel that it is already clear from the following paragraph (highlighted in blue) that, as suspected by the reviewer, only a subset of the scenes depicted a 'target item of gaze'.

Comment: Page 16: reference to Castelhano et al 2007

Action: this very useful and relevant reference is now included in the discussion.

Comment: Page 17: change word "students" 
Action: The word "Student's" here (highlighted in blue) refers to the statistician who developed these tests, and is included to prevent a capital $\mathrm{T}$ at the beginning of this sentence, which could lead to confusion with a different kind of t-test. It has therefore been left in place.

Comment: Page 22: greater consideration of neural underpinning in Discussion

Action: new sections have been added to the Discussion, on pages 23-24, addressing this issue

Comment: Page 22: include Speer et al, 2007

Action: new sections have been added to the Discussion, on pages 23-24, addressing this issue

Comment: Page 22: more discussion of finding of no difference in fixation on eye-region

Action: new sections have been added to the Discussion, on pages $23-24$, addressing this issue

Comment: Table 4: footnote contains reference to missing Table 5

Action: amended as recommended

Comment: Figure 5: label on $\mathrm{x}$-axis needed

Action: amended as recommended

Comment: Figure 2 and 3: consistency in y-axis label

Action: both Figures now have identical y-axes

We are very grateful for the two reviewers' comments and have found them extremely constructive. We have paid attention to all of the detailed and very constructive comments put forward by Reviewer 2 and hope we have addressed all of these points appropriately and thoroughly. We feel that this re-submission, with its greater emphasis on neural underpinnings of social attention and its clearer presentation of results, is now highly appropriate for publication in Neuropsychologia.

We hope that you will now agree with Reviewer 1's original evaluation and choose to publish the resubmitted work in your journal.

Yours sincerely,

The Authors 
Eye-movements reveal attention to social information in autism spectrum disorder.

Fletcher-Watson, S. ${ }^{+}$Leekam, S. R.*, Benson, V.^, Frank, M. C.\#, \& Findlay, J. M.*

$\underline{\text { Research carried out at: }}$

Durham University, Department of Psychology, Durham, UK.

Affiliations:

${ }^{+}$Newcastle University, Sir James Spence Institute, Newcastle upon Tyne, UK

* Durham University, Department of Psychology, Durham, UK

$\wedge$ University of Southampton, School of Psychology, Southampton, UK

\# Massachusetts Institute of Technology, Boston MA, USA

Corresponding Author:

Sue Fletcher-Watson, Newcastle University, Sir James Spence Institute, Royal Victoria Infirmary, Newcastle-upon-Tyne, NE1 4LP, UK.

Tel: +44 (0)1912023070

Fax: +44 (0)1912023095

Email: sue.fletcher-watson@ncl.ac.uk

Short title: Eye movements in autism.

Acknowledgements: This research was supported by a grant from the Economic and Social Research Council to SFW. 


\begin{abstract}
$\underline{\text { Abstract }}$
Autism spectrum disorder (ASD) is a neurodevelopmental condition in which children show reduced attention to social aspects of the environment. However in adults with ASD, evidence for social attentional deficits is equivocal. One problem is that many paradigms present social information in an unrealistic, isolated way. This study presented adults and adolescents, with and without ASD, with a complex social scene alongside another, non-social scene, and measured eye-movements during a three-second viewing period. Analyses first identified viewing time to different regions and then investigated some more complex issues. These were: the location of the very first fixation in a trial (indicating attentional priority); the effect of a task instruction on scan paths; the extent to which gaze-following was evident; and the degree to which participants' scan paths were influenced by the low-level properties of a scene. Results indicate a superficially normal attentional preference for social information in adults with ASD. However, more sensitive measures show that ASD does entail social attention problems across the lifespan, supporting accounts of the disorder which emphasise lifelong neurodevelopmental atypicalities. These subtle abnormalities may be sufficient to produce serious difficulties in real-life scenarios.
\end{abstract}

Key words: eye-tracking, social attention, scene viewing, preferential-looking, perception. 
Eye-movements reveal attention to social information in autism spectrum disorder.

\section{$\underline{\text { Introduction }}$}

The term autism spectrum disorder (ASD) refers to a set of neurodevelopmental disorders defined by atypicalities in three major symptom domains: communication, interaction and imagination (Wing \& Gould, 1979). Children with ASD often show significant impairments in their attention to social elements of the environment. This includes well-documented impairments in triadic joint attention (Loveland \& Landry, 1986; Leekam, Baron-Cohen, Perrett, Milders, \& Brown, 1997; Leekam, Hunnisett, \& Moore, 1998; Mundy \& Newell, 2007; Mundy, Sigman, Ungerer, \& Sherman, 1986) and attentional capture by social stimuli (Dawson, Meltzoff, Osterling, Rinaldi, \& Brown, 1998; Dawson et al., 2004). Perhaps the most fundamental aspect of social attention is attention to another person, and this has also been shown to present problems for children with autism in both interactive (Leekam, Lopez, \& Moore, 2000) and picture-viewing (Riby \& Hancock, 2008) paradigms.

The prevalence of social attention problems in autism has led many to suggest that these may be at the root of the development of the disorder (Klin, Jones, Schultz, \& Volkmar, 2003; Mundy \& Neal, 2001; Schultz, 2005). These theories all suggest that autism results from an interaction between experience and neural development, creating developmental impairments in social behaviour and the 'social brain' (Brothers, 1990; Johnson, 2005). The amygdala is particularly important in the investigation of social attention in autism, since abnormalities in this area are thought 
to be related to reduced fixation on the eye-region in autism (Dalton et al. 2005;

Corden, Chilvers \& Skuse 2008) as well as underpinning wider deficits in social cognition (Baron-Cohen et al. 1999; Baron-Cohen et al. 2000; Howard et al. 2000; Nacewicz et al. 2006).

Schultz (2005) suggests that early atypicalities in amygdala function (Dawson et al. 2002) contribute to the development of deficits in social behaviour as well as constraining neural development in regions such as the fusiform face area. This process would result in life-long neural atypicalities, affecting the developmental trajectory of people with ASD, and maintaining the social problems characteristic of autism. This interpretation is supported by evidence of functional abnormalities in the brains of adults with ASD performing social cognitive tasks (Bird, Catmur, Silani, Frith, \& Frith, 2006; Castelli, Frith, Happé, \& Frith, 2002; Critchley et al., 2000; Freitag et al., 2008; Pelphrey, Morris, \& McCarthy, 2005) and of structural abnormalities in the social brain (Frith, 2001; Kleinhans et al. 2008).

On the other hand, behavioural research shows that joint attention and social orienting is clearly related to developmental level and age in children (Leekam et al., 2000; Leekam \& Ramsden, 2006; Mundy \& Sigman, 1990; Mundy, Sigman, \& Kasari, 1994). This raises the possibility that people with ASD (particularly highfunctioning individuals) might be able to develop social skills not apparent in childhood, as they grow older. There has been very little investigation of attention to social information in adults with ASD and the question remains whether or not social attention continues to be impaired beyond childhood in autism.

One significant study to address this question employed eye-tracking technology to examine whether adults and adolescents with ASD looked at the same parts of a moving image with social content as typically-developed (TD) adults (Klin, Jones, 
Schultz, Volkmar, \& Cohen, 2002). The authors found that people with ASD had atypical fixation patterns, looking less at the eye-region and more at the mouth and objects. However, others have suggested that this multi-sensory, moving stimulus produced group differences due to complex processing requirements, which particularly affected the performance of participants with ASD (Kemner \& van Engeland, 2003). This suggestion is supported by evidence that children and adolescents with ASD only have abnormal fixation patterns for moving stimuli, which depict two people engaged in a social exchange (Speer, Cook, McMahon \& Clark 2007). The authors conclude that for static images or those which present social information in isolation (i.e. just one person) social attention is normal in autism.

Evidence from studies which present static images of isolated social information to adults with ASD remains equivocal. Some findings correspond with Speer et al, showing that fixation within a static face is normal in autism (Bar-Haim, Shulman, Lamy, \& Reuveni, 2006). Others suggest that people with ASD do fixate less on the eye-region of faces specifically (Spezio, Adolphs, Hurley, \& Piven, 2007), some that fixation to all central features of the face is reduced (Pelphrey et al., 2002). Moreover, all of these studies present only faces, without a visible body or realistic scene context, and so they tell us more about face processing (Sasson, 2006) than about attention to social information. One study that did present social information within a realistic scene showed a normal attentional bias for eye-gaze information in able young adults with autism (Fletcher-Watson, Leekam, Findlay, \& Stanton, 2008). However, the 'change blindness' method used in this case could not reveal how attention is distributed temporally and spatially to the scene as a whole.

Research measuring the eye movements of TD adults viewing social and nonsocial stimuli has recently demonstrated that they show a strong attentional preference 
for social scenes (Fletcher-Watson, Findlay, Leekam, \& Benson, 2008). TD adults are capable of identifying human figures and even faces in peripheral vision and moving their eyes directly to that spot, with no delay in eye-movement processing time. This attentional preference to look at people is increased by the introduction of a task requirement to identify the gender of the person depicted. Furthermore, there is evidence of a gaze-following process, whereby the viewer looks at the person in the scene and subsequently to the area being fixated by that person.

Replicating this study with participants with ASD can help to reveal whether they will also preferentially attend to and prioritise social information. Given the equivocal nature of existing evidence, it is hard to make concrete predictions. If stimulus complexity makes a difference to adults' attention, our static stimuli, which depict only one person, might not produce atypical fixation patterns in participants with ASD, in line with findings in children (Speer et al. 2007). On the other hand, by presenting realistic social information in a naturalistic context, our experimental stimuli could produce larger group differences than have been found in experiments using isolated face stimuli, such as that by Pelphrey and colleagues (2002). Of particular interest will be the degree to which each group fixates on the eye-region of the person depicted - a measure perhaps directly related to amygdala function (Dalton et al. 2005) and to social function (Klin et al. 2002).

In addition, we will investigate three further questions of interest for autism research. First, an examination of the effect of a task instruction on social attention will help to reveal the possible distinction between what someone with ASD does spontaneously, and what they can do when instructed. Second, the presence of gazefollowing behaviours when viewing a static visual scene will be investigated. Third, we will assess the degree to which low-level stimulus properties direct visual fixation. 
This latter question is of particular interest in the context of an ongoing debate about the relative influence of low and high-level stimulus properties on attention in TD individuals (Hayhoe \& Ballard, 2005).

Finally, some research suggests that people with ASD show atypical saccades and other types of eye-movement (Kemner, Verbaten, Cuperus, Camfferman, \& Van Engeland, 1998; Minshew, Luna, \& Sweeney, 1999; Rosenhall, Johansson, \& Gillberg, 1988; Takrae, Minshew, Luna, Krisky, \& Sweeney, 2004; Takrae, Minshew, Luna, \& Sweeney, 2004). Therefore, an initial comparison of the two participant groups in this study will also look for differences in basic eye-movement patterns.

\section{$\underline{\text { Method }}$}

\section{Participants}

The TD group comprised 15 adolescents and adults ( 2 female) aged 17-48 years, from mainstream high schools and further education colleges in the Durham area. None of these participants had taken part in previous research with this paradigm. Participants completed the Autism Quotient questionnaire to rule-out autistic spectrum diagnosis (Baron-Cohen, Wheelwright, Hill, Raste \& Plumb 2001) and all received a score well below the recommended cut-off of 32+ for ASD (TD range $10-$ 25).

The ASD group comprised 12 adolescents and adults (2 female) aged 16-23 years, with high-functioning autism or Asperger's syndrome. All these participants attended a specialist college in the Sunderland area for which a diagnosis of autism or Asperger's syndrome was a criterion of admission. All had been diagnosed by experienced clinicians (a psychiatrist or clinical psychologist employed by the 
National Health Service) working in specialised centres, as meeting DSM-IV criteria for either high-functioning autism or AS (American Psychiatric Association, 1994). These diagnoses were confirmed, upon each participant's admission to the college, by a second clinical psychologist.

The groups were group-wise matched on chronological age, rather than using one-to-one matching, since age in an adolescent and adult group is not likely to affect eye-movements or attentional preference. The full Wechsler abbreviated Scales of Intelligence (WASI) was administered to all participants (Wechsler, 1999). While the groups were comparable in terms of educational level, it was not possible to match them on IQ. There were significant group differences in full-scale IQ, verbal IQ and performance IQ. IQ scores were therefore included as covariates if there was evidence of a significant correlation with dependent variables (Keppel \& Zedeck, 1989). Descriptive statistics and t-test results for group differences are illustrated in Table 1.

[Insert Table 1 about here]

\section{Materials and Apparatus}

The stimuli consisted of 40 pairs of scenes, each pair presented in a single display, with the two scenes separated by a central, vertical black line. Each display contained one person-present (PP) scene and one person-absent (PA) scene. These scenes were paired randomly, such that a PP scene set in a living room could be paired with another from the same location-type or from another location-type (office, kitchen or garden). A sample stimulus is depicted in Figure 1. 
Stimuli were divided into two blocks of 20 combined scenes and these blocks were matched for content of the PP scene, using the following content categories: the setting of the image (garden, office, living room, kitchen); the location of the PP scene (on the left or right of the stimulus); the direction of gaze of the person depicted (into or away from the PA scene); the posture of the person depicted (sitting or standing); the fixation of the person depicted (on a visible object or off-camera); the amount of face visible; the head angle of the person depicted (level, looking up or looking down); and finally the gender of the person depicted. The person depicted in the PP scene was located randomly - in the centre of the scene, to the left or to the right - and all presented a neutral facial expression. No scenes showed someone gazing directly into the camera.

Participants saw all the stimuli, either seeing Block A in a free-viewing condition, followed by Block B in a gender-discrimination condition or vice versa. Within each block, stimuli were presented in a different random order for each participant. Stimuli were presented on a colour monitor and filled the entire screen (1024 x 768 pixels). Eye movements from one eye were recorded using a Dual Purkinje Image eye tracker as participants viewed the stimuli binocularly from a distance of 1 metre. The stimuli therefore subtended a visual angle of approximately $22^{\circ} \times 15^{\circ}$. Participants' eye movements were monitored at a rate of 200 recordings per second.

\section{Procedure}

Participants were given an information sheet outlining the study and gave their consent to be involved. A nine-point calibration was taken before each half of the experiment. The first half of the experiment was the free-viewing condition, in which no instructions were given beyond, "You are going to see some colour pictures. Just 
have a look." Each trial presentation sequence consisted of a one second blank screen followed by a one second central fixation cross and then presentation of the experimental stimulus for three seconds. Participants viewed 21 stimuli, of which the first was a practice stimulus with no data recorded.

The second half of the experiment was the gender-discrimination condition. This fixed order of conditions was selected in order to prevent any possible confound preventing genuine 'free-viewing'. Here, participants were told "You're going to see some more pictures just like before, but this time I want you to decide on the gender of the person in the picture." Trials proceeded as before but with the addition of a response screen, saying "Respond Now", which appeared after the experimental stimulus. This response screen was presented until the participant made a button-press response to indicate the perceived gender of the person in the display. Participants viewed 23 stimuli, of which the first three were practice stimuli with no data recorded. Participants were debriefed and paid $£ 10$ for their participation.

\section{$\underline{\text { Results }}$}

\section{Data preparation}

For each data set an automatic saccade detection programme detected the beginning and end of every saccade and these selections were then checked manually. This software provided an output including the path of each saccade and the location and duration of every fixation. Each stimulus presented during the experiment was divided into six domains (see Fig 1b) demarcating the Eyes, the Face-remainder and the Body of the person in the scene, the Background of each scene and the black central bar separating the pair of scenes. The Face-remainder domain consists of the 
rest of the face, excluding the Eyes domain. The tracker output was then combined with these domains to produce a data set recording the distribution of fixations across domains of interest.

Each trial lasted $3000 \mathrm{~ms}$, however due to tracker loss and the exclusion of blinks, only some of this time was recorded for some trials. Since a partial recording may not be representative, all trials in which less than $500 \mathrm{~ms}$ of data were recorded were excluded. This resulted in the exclusion of $4.25 \%$ of all trials.

There were no differences between groups in the number of excluded trials, the number of fixations recorded, the amount of time recorded, the number of lost first fixations or in accuracy to identify gender. There was also no difference in the mean number of fixations made within a trial (and consequently also the mean number of saccades), suggesting that participants had similar basic eye-movement patterns in this task. The use of percentage measures of viewing time and first fixations means that any non-significant group differences in basic oculomotor control or quantity of data recorded would be accommodated in the subsequent analysis.

\section{Viewing time in each domain}

Viewing time was calculated as the percentage of total viewing time (fixation only) recorded per trial, in each domain of interest. None of full-scale, verbal or performance IQ measures correlated with viewing time to any domain in either viewing condition (all $\mathrm{r}<.310$ and all $\mathrm{p}>.116$, with Bonferroni correction for multiple comparisons). There were also no significant correlations when these tests were repeated for each participant group separately. Therefore viewing-time scores were analysed without IQ as a covariate. Viewing time statistics for each group for each scene and domain are shown in Table 2. 
[Insert Table 2 about here]

Comparisons were made between groups for the percentage of viewing time spent in each scene and then in each domain. These comparisons investigate how attention is distributed between the two scenes, and also within sub-domains of the PP scene, across the full-viewing period of three seconds. They are designed to investigate attention to increasingly social scene regions. The analyses first compare the two scenes as a whole, then compare sub-domains of the PP scene with each other: Background vs. Person; Body vs. Face; Eyes vs. Face-remainder. The Person domain consists of the Body and Face domains, summed together and the Face domain consists of the Eyes and Face-remainder domains summed together. All ANOVAs compare a pair of domains, and also include Group (ASD vs. TD) and Condition (free-viewing vs. gender-discrimination) variables.

The first ANOVA compared viewing time between the two scenes (see Table 2), revealing no main effect, nor any interactions involving Group. There was a main effect of Scene, $\mathrm{F}(1,25)=197.59, \mathrm{p}<.001$, such that a greater percentage of viewing time was spent in the PP scene across all participants and conditions. There was also a significant interaction between Scene and Condition, $F(1,25)=41.33, \mathrm{p}<.001$. This showed that the difference in viewing time between scenes was increased in the gender-discrimination condition compared to the free-viewing condition.

To investigate viewing time within the PP scene, a comparison was made between the Background of this scene and the Person. Once more, this ANOVA revealed no group effects. There was a main effect of Domain, F $(1,25)=29.50$, $\mathrm{p}<.001$, such that a higher percentage of viewing time was spent looking at the Person 
than at the PP Background. There was also a main effect of Condition, F $(1,25)=$ $40.35, \mathrm{p}<.001$, because there were more fixations in both Background and Person domains combined, in the gender-discrimination than in the free-viewing condition. Finally, there was an interaction between Domain and Condition, F $(1,25)=73.62$, $\mathrm{p}<.001$, which showed that there was a greater difference between looking at the Person and than the Background in the gender-discrimination condition.

A comparison was also made between viewing time in the Body and Face domains, to investigate how attention was distributed between different parts of the person. This ANOVA produced a borderline main effect of Group, $F(1,25)=3.94$, $\mathrm{p}=.058$. This had a small effect size (partial $\eta^{2}=.136$ ) but represented a tendency for the ASD group to spend less viewing time on both Body and Face domains overall, compared with the TD group. There was also a main effect of Condition, F $(1,25)=$ 90.22, $\mathrm{p}<.001$, because more viewing time was spent in both the Body and Face domains in the gender-discrimination than in the free-viewing condition.

To check that the borderline group difference found here was not due to IQ differences, this analysis was repeated in an ANCOVA (type 1 sums of squares) with full-scale IQ as a covariate, which revealed no IQ effects, but a significant group difference, $\mathrm{F}(1,24)=6.19, \mathrm{p}=.02$.

Finally an ANOVA was performed on the sub domains of the Face. This was to investigate the distribution of attention to the most social element of the Person, their eyes, and to test the specific hypothesis that people with ASD look less at the eyeregion. This hypothesis was not upheld, since there were no Group effects in this analysis. There was a main effect of Condition, $\mathrm{F}(1,25)=25.34, \mathrm{p}<.001$, because, once again, more viewing time was spent in both domains in the genderdiscrimination than in the free-viewing condition. There was also a main effect of 
Domain, $\mathrm{F}(1,25)=27.41, \mathrm{p}<.001$, such that more time was spent viewing the rest of the face than the eye-region specifically.

This initial analysis of viewing times in different domains reveals no significant group differences, and so a series of analyses controlling for domain size were performed, to look for more subtle group differences in the spatial distribution of attention.

Viewing time, adjusted according to domain size

Each stimulus was divided into six domains for analysis (as depicted in Figure 1b). The domains varied in size between images, and in particular, Face-remainder and Eyes domains were always much smaller than any other domain. Therefore viewing time scores were adjusted to give domain-relative measures. Viewing times as percentages were divided by the size of the relevant domain, also expressed as a percentage of the total stimulus size. If fixations were spread randomly across the stimulus, one would expect a score close to one (e.g. 50\% of fixation time / $50 \%$ of stimulus size). If fixations were being directed to a domain more than randomly predicted, this score would be greater than one, and vice versa. Domain-relative viewing times are reported in Table 3.

[Insert Table 3 about here]

A series of t-tests were conducted for each group separately, investigating whether scores differed significantly from one. In order to reduce the number of ttests required, this analysis was only performed on data from the free-viewing condition. Because 12 t-tests were being performed, (2 groups x 6 domains), a 
Bonferroni adjustment was made such that a p-value of .004 or less was required for significance. Both groups showed a domain-relative score significantly different from one (all p<.004) for every domain (PP Background, Person, Body, Face, FaceRemainder) except the Eyes domain. Viewing time to the Eyes domain did not significantly differ from one in either participant group, indicating that this region was viewed only as much as would be expected by chance. For the PP Background, domain-relative viewing times were less than one, indicating that this domain was fixated less than would be predicted by a random viewing pattern. The other four domains were all fixated more than would be predicted by a random viewing pattern.

Domain-relative viewing time for the background of the PP Scene did not significantly differ from that for the entire PA Scene, for either group or viewing condition (all p>.10), indicating that differences in viewing times between these two scenes is due to the time spent looking at the person depicted.

\section{Summary of Viewing Time Analysis}

The viewing time data reveal a consistent pattern of results. First, participants from both groups show a preference to look at social elements of the display: the PP scene is viewed more than the PA scene and the Person is viewed more than the Background of the PP scene. These biases are consistent across individual participants, and exaggerated in the gender-discrimination condition.

The Body and Face receive an equal proportion of viewing time, though the TD participants tend to look at both domains more than the ASD group. This is the only group effect in the viewing time data and it is only marginally significant $(\mathrm{p}=.058)$. Both Body and Face receive more viewing time in the gender-discrimination condition than in the free-viewing condition and across both conditions, the Eyes are 
looked at for less time than the rest of the face. While the eyes are often thought of as the most socially informative region of the face, this result emphasises the sociocommunicative importance of the mouth area.

Domain-relative scores show that all domains are more likely to be fixated than would be predicted by chance, except for the Eyes, the background of the PP scene and the entire PA scene. There is no difference in fixation probability between these two latter domains, indicating that the attentional preference for the PP scene is entirely driven by fixations on the Person in the scene.

In conclusion therefore, these analyses indicate no group differences in the allocation of fixations to different areas of a naturalistic, social and non-social display over time.

\section{First fixations}

The first fixation is defined as that fixation following the first saccade made after trial-onset. This measure examines which items received attentional priority in the scene, rather than simply which items received the greatest amount of viewing time. Data on the distribution of first fixations are shown in Table 4.

None of the IQ measures (full-scale, verbal or IQ) correlated with the percentage of first fixations made any domain in either viewing condition (all Pearson's $r<.304$, and all p>.124, with Bonferroni correction for multiple tests) and this lack of significant results holds when the participant groups are analysed separately. Nor were there any group differences in the latency of first saccades to each domain (all p>.057, Bonferroni adjustment requires $\mathrm{p}<.0035$ for significance). Therefore no IQ measures were included in the analyses of first fixations. As in the analysis of viewing 
times, a series of ANOVAs was used to compare the percentage of first fixations made in each Scene and then pairs of domains within the PP scene.

[Insert Table 4 about here]

An ANOVA comparing first fixations in the two scenes revealed a Scene by Group interaction, $F(1,25)=5.41, \mathrm{p}=.028$, which showed that the TD group had a larger bias to make first fixations on the PP Scene than the ASD group (see Fig 2). Post-hoc t-tests (both viewing conditions' data combined) reveal that the ASD group made fewer PP scene first fixations, $\mathrm{t}(25)=2.51, \mathrm{p}=.019$, and more PA scene first fixations, $\mathrm{t}$ $(25)=2.00, p=.057$, than the TD group. However, both groups did have a bias to make a higher proportion of first fixations in the PP scene, despite the difference in degree of bias: TD group $\mathrm{t}(14)=22.15, \mathrm{p}<.001 ;$ ASD group $\mathrm{t}(11)=9.44, \mathrm{p}<.001$.

This ANOVA also produced a main effect of Scene, $\mathrm{F}(1,25)=394.73, \mathrm{p}<.001$, indicating that a larger percentage of first fixations fell in the PP than the PA Scene. There was also a Scene by Condition interaction, $F(1,25)=21.39, \mathrm{p}<.001$, showing that the difference in the distribution of first fixations between scenes was greater in the gender-discrimination condition.

[Insert Figure 2 about here]

An ANOVA on first fixations in the PP Background and Person domains produced a main effect of Group, $\mathrm{F}(1,25)=6.31, \mathrm{p}=.019$, because the participants with ASD made a smaller percentage of first fixations in both these PP scene domains combined. In addition, there was a Group by Domain interaction, F $(1,25)=10.23$, 
$\mathrm{p}=.004$, showing that the TD group had a strong bias to make more first fixations on the Person than the PP Background, but that this bias was reduced in the ASD group (see Fig 3). Post-hoc t-tests revealed that in fact the preference present in the TD group fails to reach significance for the ASD group: TD group $t(14)=6.87, \mathrm{p}<.001$; ASD group $\mathrm{t}(11)=1.98, \mathrm{p}=.073$. Main effects of Condition, $\mathrm{F}(1,25)=17.77$, $\mathrm{p}<.001$, and of Domain, $\mathrm{F}(1,25)=22.65, \mathrm{p}<.001$, were also found, showing a larger percentage of first fixations in the Person than the Background, and in the genderdiscrimination condition.

[Insert Figure 3 about here]

An ANOVA also compared the two domains within the Person (Body and Face). Again, there was a main effect of Group, $\mathrm{F}(1,25)=11.52$, $\mathrm{p}=.002$, showing that the ASD group made fewer first fixations to the Person than the TD group. There were also main effects of Condition, $\mathrm{F}(1,25)=8.15, \mathrm{p}=.009$, and Domain, $\mathrm{F}(1,25)=$ 23.51, $\mathrm{p}<.001$, showing a larger proportion of first fixations in the Body than the Face, and in the gender-discrimination condition.

A final ANOVA compared the two face domains (Eyes and Face-remainder), revealing a main effect of Group, $F(1,25)=11.92$, $\mathrm{p}=.002$, showing again that the ASD group made fewer first fixations than the TD group to the face as a whole. There was also an interaction of Group and Domain, $\mathrm{F}(1,25)=15.98, \mathrm{p}<.001$, which resulted from both groups making a similar percentage of first fixations in the Eyes domain, while the TD group made a much larger percentage of first fixations to the rest of the face (see Table 4). Post-hoc t-tests showed that in this case, both groups did show a significant bias to look at the rest of the face more than the eye-region: TD 
group $\mathrm{t}(14)=8.27, \mathrm{p}<.001 ;$ ASD group $\mathrm{t}(11)=5.51, \mathrm{p}<.001$. Finally, there was also a main effect of Domain, $\mathrm{F}(1,25)=73.40, \mathrm{p}<.001$, indicating that a smaller percentage of first fixations were made to the eye-region than to the rest of the face. There was no effect of Condition in this ANOVA, indicating that the genderdiscrimination instruction did not effect viewing time to Face regions.

\section{Looking where others look}

Two new domains were also created (see Fig 1c and 1d), in order to investigate the secondary question of whether participants looked at the areas looked at by the person represented in the scene. The Viewing Cone domain was a $30^{\circ}$ cone extending from the centre of the eye-region of the person depicted. Thirty degrees is the range visible to an individual without moving their head (Sanders, 1963). The cone therefore encompassed the entire region visible to the person depicted in the scene, even in cases where it was not possible to identify eye-gaze direction (e.g. Fig 1). The area was selected using the cues of head-angle and, where available, eye-gaze direction (which were always congruent), and a $30^{\circ}$ cone was drawn over the scene using Paint Shop Pro.

In addition, for the subset of scenes (26/40) in which a visible object was being fixated by the person in the scene, this 'object' was analysed separately with its own domain. There were no correlations between any IQ score and viewing time to these two domains in either viewing condition or group (all $r<.353$, all $\mathrm{p}>.157$ ). Therefore IQ was not included in these analyses.

Domain relative fixation time scores were calculated as before: a score of one indicates randomly allocated viewing, less than one indicates a paucity of viewing of this area and scores greater than one indicate above chance viewing in this area. 
Student's t-tests showed that looking at the Viewing-Cone did not significantly differ from one, across both Groups and Conditions, indicating that this region was fixated neither more nor less than a random viewing pattern would predict. The Object domain was also not fixated more than randomly predicted for the ASD group in both conditions. However the TD group did fixate the Object more than expected, $\mathrm{t}(14)=$ $3.36, p=.005$, in the free-viewing condition only. This result was confirmed by a t-test showing a significant difference between groups for domain-relative viewing time in the Object in the free-viewing condition, $\mathrm{t}(20.15)=2.57, \mathrm{p}=.018$ (equal variances not assumed, $\mathrm{F}=7.73, \mathrm{p}=.01$ ). This result suggests that in the free-viewing condition the TD group were more inclined to look at the Object being looked at by the Person in the scene than the ASD group.

Low-level image feature analysis

In a further analysis, we attempted to test whether the ASD and TD groups differed in the degree to which their eye-gaze was attracted by low-level features of the stimuli (similar to the approach taken with infant data by Frank, Vul, \& Johnson, submitted). To do this, we created saliency maps (Itti \& Koch, 2001) - maps of the low-level visual properties of the stimulus pictures - to quantify what locations in the images would attract participants' attention in the free-viewing condition.

We reasoned that an analysis of the viewing patterns of the participants relative to these saliency maps might reveal subtle differences in the degree to which ASD and TD participants' gaze was drawn by low-level, bottom-up properties of the stimuli. Note that this analysis is complementary to, rather than at odds with, the region-of-interest-based analyses we have used elsewhere in the paper. For instance, it might be the case that while both groups looked equally at a particular domain, one or 
the other population might be drawn differentially to more salient regions within that domain.

[Insert Figure 4 about here]

We constructed these low-level maps using difference-of-Gaussians filters to calculate luminance contrast, orientation contrast, red-green and blue-yellow contrast, and colour saturation (an example showing luminance contrast is given in Figure 4). We then computed how well each saliency map was able to predict the eyemovements of the participants in each group. For each map, rather than identifying fixations and saccades (as in the other analyses reported here), we instead calculated the mean likelihood of each participants' scan-path by averaging the likelihood of each point along that path at each sample recorded by the eye-tracker. This mean likelihood measure gives us a way of comparing ASD and TD participants on the degree to which low-level image features predict their attentional allocation.

We applied the saliency map analysis to data from both the free-viewing and gender conditions, and analyzed the resulting mean likelihoods in an ANOVA with Condition, Group, and Feature (luminance, orientation, red-green, blue-yellow, and saturation) as factors. We found a significant main effect of Feature, $\mathrm{F}(4,253)=$ 63.19, $\mathrm{p}<.0001$, but no effects of either Condition $(\mathrm{p}=.25)$ or Group $(\mathrm{p}=.86)$ and no interactions (all $\mathrm{p}$ values $>.56$ ).

Orientation contrast was the best predictor of gaze, with luminance, red-green, and blue-yellow contrast all approximately equivalent, and saturation the worst predictor. However, no map predicted the gaze of one group differentially, suggesting 
that ASD participants' fixations were no more predictable as a function of low-level stimulus information than were the fixations of TD participants (see Figure 5).

[Insert Figure 5 about here]

\section{Summary}

The first goal of this analysis was to investigate attentional preferences for social information in people with and without ASD. All analyses show a strong bias for both groups to look at the person-present scene and particularly the Person themselves. For the TD group, this bias appears in the first fixation. The ASD group differ from the TD group only at a subtle level: a preference for social information at the first fixation is either less strong, or absent, in participants with ASD, indicating a reduced attentional priority for social elements of a scene.

Both groups respond to an instruction to discriminate gender by increasing fixation on the Person depicted. The ASD group do not show spontaneous gazefollowing in their fixation of the Object domain in the free-viewing condition; a behaviour which is present in the TD group. Both groups also make fixations which are not highly predictable by the low-level visual properties of the stimulus.

\section{Discussion}

The current study used a novel preferential-looking task to investigate how the attention of adults with and without ASD is distributed across social and non-social scenes, and social and non-social elements within a scene. The task was combined with an eye-tracking methodology, which provides an on-line measure of visual and 
cognitive processing of the material under inspection (Liversedge \& Findlay, 2000). Moreover, the method assessed spontaneous, naturalistic attention, without making extra processing demands upon participants. It was predicted that this study would reveal a reduced social attentional bias in people with ASD compared to their TD peers, but this prediction was only upheld for first fixation measures of attentional priority for social stimuli.

The TD group show a strong bias to fixate the person-present scene, and particularly the Person themselves, replicating the findings of a previous study using the same method (Fletcher-Watson et al., 2008). On a gross level, the ASD group show a similar viewing pattern to their TD peers, also preferentially looking at the person-present, rather than the person-absent scene. Likewise, an instruction to identify the person's gender increases viewing of the person-present scene and particularly of the relevant person-domains, from the first fixation in both groups. This indicates that the participants with ASD involved in this study did not show any aversion to social information (Dalton et al., 2005; Kylliainen \& Hietanen, 2006), though the stimuli also did not feature direct gaze.

The eye-region of the face is thought to convey a great deal of social information and attention to this area has been extensively studied in people with ASD. Evidence from children and adults has been found showing reduced attention to eyes (Klin et al. 2002; Pelphrey et al. 2002; Dalton et al. 2005; Spezio et al. 2007; Corden et al. 2008; Riby and Hancock 2008) but a minority of studies report normal attention to this area (van der Geest et al. 2002; Bar-Haim et al. 2006; Fletcher-Watson et al. 2008). The difference may in part be due to differences in stimulus type (Speer et al. 2007) but this is not the whole story since studies recording eye-movements to static, isolated face stimuli have produced opposing results (e.g. Pelphrey et al, 2002 vs. van der 
Geest at al. 2002). Our finding contributes to this controversial literature by revealing that adults and adolescents with ASD do spontaneously attend to the eye-region of a person, presented in a static but naturalistic image.

This finding is particularly important in the context of current neurodevelopmental hypotheses of autism, which suggest that childhood abnormalities in amygdala function have cascading effects on the development of the social brain and behaviour (Schultz 2005). Abnormalities of the amygdala are proposed to play a particularly significant role in deficits in attending to the eyeregion of faces, and interpreting emotions and mental states from this cue (Ashwin et al. 2006; Corden et al. 2008). Another suggestion is that the relationship between amygdala dysfunction and reduced fixation on eyes is mediated by a heightened emotional response to direct gaze in particular (Dalton et al. 2005). It is therefore possible that our finding of normal attention to the eye-region was due to our choice of stimuli, none of which featured direct gaze to camera or emotional content. On the other hand, other studies have found that people with ASD do spontaneously use the eye-region to interpret complex emotional states from faces with direct gaze (Back et al. 2007).

The analyses reviewed so far suggest that attention to social information is normal in adults and adolescents with ASD. They imply that it is possible for people with ASD to develop social attentional skills with age and developmental level, perhaps despite underlying atypicalities in the social brain. However, further investigation reveals that, in the ASD group, looking at a social stimulus is consistently less marked at the first fixation. This subtle, but consistent and significant finding reinforces the suggestion that autism is a lifelong disorder, in which neurodevelopmental difficulties in childhood produce long-term effects. These group 
differences do not appear to be driven by variation in IQ in this sample. Nevertheless, taking intellectual and developmental level and age into account in future studies of autism must persist. For example, these results can be contrasted with a social sceneviewing study which demonstrated significantly reduced attention to people in a lower-functioning and younger group of children with ASD, compared with controls (Riby \& Hancock, 2008).

While the difference between our groups in priority given to social information is subtle, it is possible that the consequences in the real world could be serious. Social information is often presented fleetingly as people's speech, facial expression and gestures change rapidly from moment to moment. Any impairment in attending to or keeping track of this shifting array of social input would be effective in derailing attempts to interact with other people. Thus the fine attentional differences apparent here could translate into more significant social problems for people with ASD in every day life. This might explain the finding that dynamic stimuli present particular problems for people with ASD in terms of their ability to attend to relevant social cues (Klin, Jones, Schultz, Volkmar, \& Cohen, 2002; Speer et al. 2007). Likewise processing multi-sensory information may present a particular challenge to people with ASD (Iarocci \& McDonald, 2006).

TD participants show a tendency to look at the object being fixated by the person in the scene, in the free-viewing condition only, in line with previous findings (Castelhano et al. 2007). Since the eye-region was not fixated more than would be predicted by a random pattern of eye-movements, this finding raises the possibility that participants were using other cues such as head angle and body posture to identify the target of the depicted individual's gaze, as has been shown elsewhere (Langton, 2000). The ASD group do not show evidence of following these social cues, 
suggesting that the drive to share attention is absent or weak in this group, despite their age and high-functioning status. Joint attention has rarely been studied among adults with ASD but this finding provides evidence that a difficulty with sharing attention may also persist across the lifespan.

The low-level saliency map analysis of fixation distribution showed that both groups were equally unlikely to have their attention affected by low-level stimulus features such as contrast, brightness and colour. This analysis is important in demonstrating that the perceptual basis of the social attentional abilities seen in our participants with ASD is normal. Often, participants with ASD show abilities which, on further inspection, seem to be based on an atypical strategy, but in this experiment there is no evidence that the fixations of participants with ASD are atypically affected by low-level stimulus features.

\section{Conclusions}

The eye-movement measures obtained from this preferential-looking task illustrate subtle differences in the distribution of attention to social information between adults with and without ASD. Even in the high-functioning autistic sample who took part in this study, there is evidence of social attention atypicalities, with less priority for social information and reduced gaze-following. This supports the suggestion that the reciprocal relationship between abnormal social experience and brain development in childhood results in long-lasting impairments, which maintain deficits in social cognition in people with ASD into adulthood. This work demonstrates how important it is to investigate autism across the lifespan and not just in children. 
Nevertheless, within a social stimulus (namely a person) both TD and ASD participants showed similar distribution of attention between different socially informative regions. These results emphasise the need to investigate attention to different kinds of social information, presented in a realistic context (Smilek, Birmingham, Cameron, Bischof, \& Kingstone, 2006). Future studies should focus on systematically varying both the level of social content present (from pictures of isolated faces up to real human beings) and the complexity of that information (e.g. motion, multi-sensory qualities, affective content). It is possible that presenting emotionally neutral stimuli without direct gaze could help to overcome the difficulty people with ASD have in attending to the eyes. 
Table 1: Descriptive statistics for each group, with t-test results for group comparisons

\begin{tabular}{llllll} 
& ASD & \multicolumn{5}{l}{ TD } \\
\hline & Mean & SD & Mean & SD & t-test result \\
\hline Age (years) & 18.8 & 2.3 & 21.5 & 7.8 & $\mathrm{t}(25)=1.19, \mathrm{p}=.24$ \\
FSIQ & 91.3 & 12.7 & 107.1 & 13.5 & $\mathrm{t}(25)=3.06, \mathrm{p}=.005$ \\
VIQ & 87.2 & 12.3 & 104.5 & 13.0 & $\mathrm{t}(25)=3.53, \mathrm{p}=.002$ \\
PIQ & 97.3 & 15.9 & 108.7 & 13.5 & $\mathrm{t}(25)=2.02, \mathrm{p}=.054$
\end{tabular}


Table 2: Percentage of total viewing time in each scene and domain, by group and viewing condition (means with SDs in brackets).

\begin{tabular}{ll|ll|llll} 
& & PA Scene & PP Scene & $\begin{array}{l}\text { PP } \\
\text { Background }\end{array}$ & & Body & \multicolumn{2}{l}{$\begin{array}{l}\text { Face- } \\
\text { remainder }\end{array}$} & Eyes \\
\hline Free & ASD & $38.1(7.0)$ & $55.4(7.6)$ & $28.2(7.0)$ & $13.3(5.3)$ & $9.6(11)$ & $4.3(5.9)$ \\
& TD & $37.2(8.9)$ & $58.9(9.4)$ & $25.9(6.8)$ & $15.8(5.6)$ & $11.9(9.6)$ & $5.3(5.6)$ \\
\hline Gender & ASD & $23.5(9.6)$ & $71.2(11)$ & $24.0(8.0)$ & $23.2(8.9)$ & $17.2(10)$ & $6.8(5.2)$ \\
& TD & $19.6(12)$ & $77.2(11)$ & $19.8(9.8)$ & $28.2(9.7)$ & $19.7(11)$ & $9.5(9.1)$ \\
\hline
\end{tabular}

NB: Percentages for the Person-Absent and Person-Present scenes do not sum to $100 \%$ because a small proportion of viewing time was spent in the central bar dividing the two scenes (see Fig 1). Standard deviations for viewing time in the Faceremainder and Eyes domains are high, because these domains are so variable in size. Faces ranged from $.09 \%$ to $1.65 \%$ of the display, while Eyes took up between $.01 \%$ and $.24 \%$ of the display. Consequently, the proportion of time fixating these domains was also highly variable. 
Table 3: Domain-relative viewing time by group and viewing condition, for domains $\underline{\text { within the person-present scene (means with SDs in brackets) }}$

\begin{tabular}{llllll}
\cline { 3 - 6 } & & PP Background & Body & Face-remainder & Eyes \\
\hline Free & ASD & $.68(.17)$ & $2.27(.78)$ & $58.9(54)$ & $68.1(75)$ \\
& TD & $.62(.17)$ & $2.84(1.0)$ & $68.6(45)$ & $110.3(137)$ \\
\hline Gender & ASD & $.58(.19)$ & $4.79(2.0)$ & $112.3(71)$ & $148.4(118)$ \\
& TD & $.47(.23)$ & $5.53(2.3)$ & $111.1(65)$ & $164.0(164)$ \\
\hline
\end{tabular}


Table 4: Percentage of first fixations in each scene and domain, by group and viewing condition (means with SDs in brackets).

\begin{tabular}{ll|ll|llll} 
& & PA Scene & PP Scene & $\begin{array}{l}\text { PP } \\
\text { Background }\end{array}$ & & $\begin{array}{l}\text { Face- } \\
\text { remainder }\end{array}$ & Eyes \\
\hline Free & ASD & $21.4(13)$ & $73.7(14)$ & $37.3(12)$ & $25.0(10)$ & $8.6(9.1)$ & $2.7(4.3)$ \\
& TD & $15.0(9.6)$ & $81.7(12)$ & $23.3(10)$ & $35.2(9.1)$ & $21.2(19)$ & $2.1(4.7)$ \\
\hline Gender & ASD & $13.7(9.8)$ & $81.2(12)$ & $32.7(12)$ & $36.3(15)$ & $10.5(8.2)$ & $1.8(2.7)$ \\
& TD & $7.2(4.4)$ & $92.1(5.2)$ & $25.7(17)$ & $37.1(14)$ & $25.4(12)$ & $4.0(4.3)$
\end{tabular}

NB: In Table 4, percentages for the Person-Present and Person-Absent scenes do not sum to $100 \%$ because a small number of first fixations were made in the central bar dividing the two scenes. 
Figure 1: A sample stimulus, illustrating the domains used in analyses

Figure 2: Interaction between percentage of first fixations in each Scene with Group

Figure 3: Interaction of percentage of first fixations in two Domains (PP background and Person) with Group, across both viewing conditions.

Figure 4. Example of a low-level saliency map using difference-of-Gaussians filters to $\underline{\text { calculate luminance contrast }}$

Figure 5. Mean likelihood of fixation in the free-viewing condition, plotted by group and by low-level image feature.

NB: Each saliency map constitutes a set of quantitative predictions about which areas of the images are salient to participants. Absolute likelihoods are very small since they represent the probability of the point of fixation falling in the precise location it did rather than any other location on the image. Although there were significant differences between the mean likelihoods of different feature maps, ASD and TD participants did not differ in the predictability of their looking with respect to saliency. 


\section{$\underline{\text { References }}$}

Ashwin, C., S. Wheelwright, et al. (2006). "Finding a face in the crowd: Testing the anger superiority effect in Asperger Syndrome." Brain and Cognition 61(1): 78-95.

Back, E., Ropar, D., \& Mitchell, P. (2007). Do the eyes have it? Inferring mental states from animated faces in autism. Child Development, 78(2), 397-411.

Bar-Haim, Y., Shulman, C., Lamy, D., \& Reuveni, A. (2006). Attention to eyes and mouth in high-functioning children with autism. Journal of Autism and Developmental Disorders, 36(1), 131-137.

Baron-Cohen, S., H. A. Ring, et al. (2000). "The amygdala theory of autism." Neuroscience \& Biobehavioral Reviews 24(3): 355-364.

Baron-Cohen, S., H. A. Ring, et al. (1999). "Social intelligence in the normal and autistic brain: An fMRI study. ." European Journal of Neuroscience 11: 18911898.

Baron-Cohen, S., S. Wheelwright, et al. (2001). "The Autism-Spectrum Quotient (AQ): Evidence from Asperger syndrome/high-functioning autism, males and females, scientists and mathematicians " Journal of Autism and Developmental Disorders 31(1): 5-17.

Bird, G., Catmur, C., Silani, G., Frith, C., \& Frith, U. (2006). Attention does not modulate responses to social stimuli in autism spectrum disorders. Neuroimage, 31, 1614-1624.

Brothers, L. (1990). The social brain: A project for integrating primate behaviour and neurophysiology in a new domain. Concepts in Neuroscience, 1, 27-51.

Castelhano, M., M. Wieth, et al. (2007). I See What You See: Eye Movements in Real-World Scenes Are Affected by Perceived Direction of Gaze. Attention in Cognitive Systems. Theories and Systems from an Interdisciplinary Viewpoint: $251-262$.

Castelli, F., Frith, C., Happé, F., \& Frith, U. (2002). Autism, Asperger syndrome and brain mechanisms for the attribution of mental states to animated shapes. Brain 125, 1839-1849. 
Corden, B., R. Chilvers, et al. (2008). "Avoidance of emotionally arousing stimuli predicts social-perceptual impairment in Asperger's syndrome." Neuropsychologia 46: 137-147.

Critchley, H. D., Daly, E. M., Bullmore, E. T., Williams, S. C., van Amelsvoort, T., Robertson, D. M., et al. (2000). The functional neuroanatomy of social behaviour: Changes in cerebral blood flow when people with autistic disorder process facial expressions. Brain, 123(Pt 11), 2203-2212.

Dalton, K. M., Nacewicz, B. M., Johnstone, T., Schaefer, H. S., Gernsbacher, M. A., Goldsmith, H. H., et al. (2005). Gaze fixation and the neural circuitry of face processing in autism. Nature Neuroscience, 8(4), 519-526.

Dawson, G., L. Carver, et al. (2002). "Neural correlates of face and object recognition in young children with autism spectrum disorder, developmental delay and typical development." Child Development 73: 700-717

Dawson, G., Meltzoff, A. N., Osterling, J., Rinaldi, J., \& Brown, E. (1998). Children with autism fail to orient to naturally occurring social stimuli. Journal of Autism and Developmental Disorders, 28(6), 479-485.

Dawson, G., Toth, K., Abbott, R., Osterling, J., Munson, J., Estes, A., et al. (2004). Early social attention impairments in autism: Social orienting, joint attention and attention to distress. Developmental Psychology, 40(2), 271-283.

Fletcher-Watson, S., Findlay, J. M., Leekam, S. R., \& Benson, V. (2008). Rapid detection of person information in a naturalistic scene. Perception, e-pub.

Fletcher-Watson, S., Leekam, S. R., Findlay, J. M., \& Stanton, E. C. (2008). Brief Report: Young adults with autism spectrum disorder show normal attention to eye-gaze information: Evidence from a new change blindness paradigm. Journal of Autism and Developmental Disorders, e-pub.

Frank, M. C., Vul, E., \& Johnson, S. P. (submitted). Development of infants' face preference during the first year. Cognition.

Freitag, C. M., Konrad, C., Haberlen, M., Kleser, C., von Gontard, A., Reith, W., et al. (2008). Perception of biological motion in autism spectrum disorders. Neuropsychologia, e-pub.

Frith, U. (2001). Mind blindness and the brain in autism. Neuron 32, 969-979.

Hayhoe, M., \& Ballard, D. (2005). Eye movements in natural behaviour. Trends in Cognitive Sciences, 9(4), 188-194. 
Howard, M. A., P. E. Cowell, et al. (2000). "Convergent neuroanatomical and behavioural evidence of an amygdala hypothesis of autism." Neuroreport 11: 2931-2935.

Iarocci, G., \& McDonald, J. (2006). Sensory integration and the perceptual experience of persons with autism. Journal of Autism and Developmental Disorders, 36(1), 77-89.

Itti, L., \& Koch, C. (2001). Computational modeling of visual attention. Nature Reviews Neuroscience, 2(3), 194-203.

Johnson, M. H. (2005). Developmental Cognitive Neuroscience. Oxford: Blackwell.

Kemner, C., \& van Engeland, H. (2003). Autism and Visual Fixation. American Journal of Psychiatry, 160(7), 1358-1359.

Kemner, C., Verbaten, M. N., Cuperus, J. M., Camfferman, G., \& Van Engeland, H. (1998). Abnormal saccadic eye movements in autistic children. Journal of Autism and Developmental Disorders, 28(1), 61-67.

Keppel, G., \& Zedeck, S. (1989). Data analysis for research designs. London: W. H. Freeman and Co.

Kleinhans, N. M., Richards, T., Sterling, L., Stegbauer, K. C., Mahurin, R., Johnson, L. C., et al. (2008). Abnormal functional connectivity in autism spectrum disorders during face processing. Brain, 131, 1000-1012.

Klin, A., Jones, W., Schultz, R., \& Volkmar, F. (2003). The enactive mind or from actions to cognition: lessons from autism. Philosophical Transactions of the Royal Society, B, 358, 345-360.

Klin, A., Jones, W., Schultz, R., Volkmar, F., \& Cohen, D. (2002). Visual fixation patterns during viewing of naturalistic social situations as predictors of social competence in individuals with autism. Archives of general psychiatry, 59, 809-815.

Kylliainen, A., \& Hietanen, J. K. (2006). Skin conductance responses to another person's gaze in children with autism. Journal of Autism and Developmental Disorders, 36(4), 517-525.

Langton, S. R. H. (2000). The mutual influence of gaze and head orientation in the analysis of social attention direction. Quarterly Journal of Experimental Psychology, 53A(3), 825-845. 
Leekam, S. R., Baron-Cohen, S., Perrett, D., Milders, M., \& Brown, S. (1997). Eyedirection detection: A dissociation between geometric and joint attention skills in autism. British Journal of Developmental Psychology, 15, 77-95.

Leekam, S. R., Hunnisett, E., \& Moore, C. (1998). Targets and cues: Gaze following in children with autism. Journal of Child Psychology and Psychiatry and Allied Disciplines, 39(7), 951-962.

Leekam, S. R., Lopez, B., \& Moore, C. (2000). Attention and joint attention in preschool children with autism. Developmental Psychology, 36(2), 261-273.

Leekam, S. R., \& Ramsden, C. A. H. (2006). Dyadic orienting and joint attention in preschool children with autism. Journal of Autism and Developmental Disorders, 36(2), 185-198.

Liversedge, S. P., \& Findlay, J. M. (2000). Saccadic eye movements and cognition. Trends in Cognitive Sciences, 4(1), 6-14.

Loveland, K., \& Landry, S. (1986). Joint attention and language in autism and developmental language delay. Journal of Autism and Developmental Disorders, 16, 335-349.

Milne, E., Swettenham, J., \& Campbell, R. (2005). Motion perception and autistic spectrum disorder: A review. Current Psychology of Cognition, 23(1), 3-36.

Minshew, N., Turner, C. A., \& Goldstein, G. (2005). The application of short forms of the Wechsler intelligence scales in adults and children with high-functioning autism. Journal of Autism and Developmental Disorders, 35(1), 45-52.

Minshew, N. A., Luna, B., \& Sweeney, J. A. (1999). Oculomotor evidence for neocortical systems but not cerebellar dysfunction in autism. Neurology, 52, 917-922.

Mundy, P., \& Neal, A. R. (2001). Neural plasticity, joint attention and a transactional social-orienting model of autism. International Review of Research in Mental Retardation, 23, 139-168.

Mundy, P., \& Newell, L. C. (2007). Attention, joint attention and social cognition. Current Directions in Psychological Science, 16(5), 269-274.

Mundy, P., \& Sigman, M. (1990). A longitudinal study of joint attention and language development in autistic children. Journal of Autism and Developmental Disorders, 20(1), 115-128. 
Mundy, P., Sigman, M., \& Kasari, C. (1994). Joint attention, developmental level and symptom presentation in autism. Development and Psychopathology, 6(3), $389-401$.

Mundy, P., Sigman, M., Ungerer, J., \& Sherman, T. (1986). Defining social deficits of autism: The contribution of non-verbal communication measures. Journal of Child Psychology and Psychiatry and Allied Disciplines, 27, 657-659.

Nacewicz, B. M., Dalton, K. M., Johnstone, T., Long, M. T., McAuliff, E. M., Oakes, T. R., et al. (2006). Amygdala volume and nonverbal social impairment in adolescent and adult males with autism. Archives of general psychiatry, 63, 1417-1428.

Pelphrey, K. A., Sasson, N., Reznick, J. S., Paul, G., Goldman, B., \& Piven, J. (2002). Visual scanning of faces in autism. Journal of Autism and Developmental Disorders, 32, 249-261.

Pelphrey, K. A., Morris, J. A., \& McCarthy, G. (2005). Neural basis of eye gaze processing deficits in autism. Brain.

Riby, D., \& Hancock, P. J. B. (2008). Research note: viewing it differently: social scene perception in Williams syndrome and autism Neuropsychologia, in press.

Rosenhall, U., Johansson, E., \& Gillberg, C. (1988). Oculomotor findings in autistic children. The Journal of Laryngology and Otology, 102(5), 435-439.

Sasson, N. (2006). The development of face processing in autism. Journal of Autism and Developmental Disorders, 36(3), 381-394.

Schultz, R. T. (2005). Developmental deficits in social perception in autism: the role of the amygdala and fusiform face area. International Journal of Developmental Neuroscience, 23, 125-141.

Smilek, D., Birmingham, E., Cameron, D., Bischof, W., \& Kingstone, A. (2006). Cognitive ethology and exploring attention in real-world scenes. Brain Research, 1080, 101-119.

Speer, L. L., A. E. Cook, et al. (2007). "Face processing in children with autism: Effects of stimulus contents and type." Autism 11(3): 265-277.

Spezio, M. L., Adolphs, R., Hurley, R. S. E., \& Piven, J. (2007). Analysis of face gaze in autism using "Bubbles". Neuropsychologia, 45, 144-151.

Takarae, Y., Minshew, N., Luna, B., Krisky, C. M., \& Sweeney, J. A. (2004). Pursuit eye-movement deficits in autism. Brain, 127(Pt 12), 2584-2594. 
Takarae, Y., Minshew, N., Luna, B., \& Sweeney, J. A. (2004). Oculomotor abnormalities parallel cerebellar histopathology in autism. Journal of Neurology, Neurosurgery and Psychiatry, 75(9), 1359-1361.

van der Geest, J. N., C. Kemner, et al. (2002). "Gaze behaviour of children with pervasive developmental disorder towards human faces: a fixation time study." Journal of Child Psychology and Psychiatry and Allied Disciplines 43(5): 669-678.

Wechsler, D. (1999). Wechsler abbreviated Scales of Intelligence (WASI). USA: Psychological Corporation.

Wing, L., \& Gould, J. (1979). Severe impairments of social interaction and associated abnormalities in children: epidemiology and classification. Journal of Autism and Developmental Disorders, 9, 11-29. 


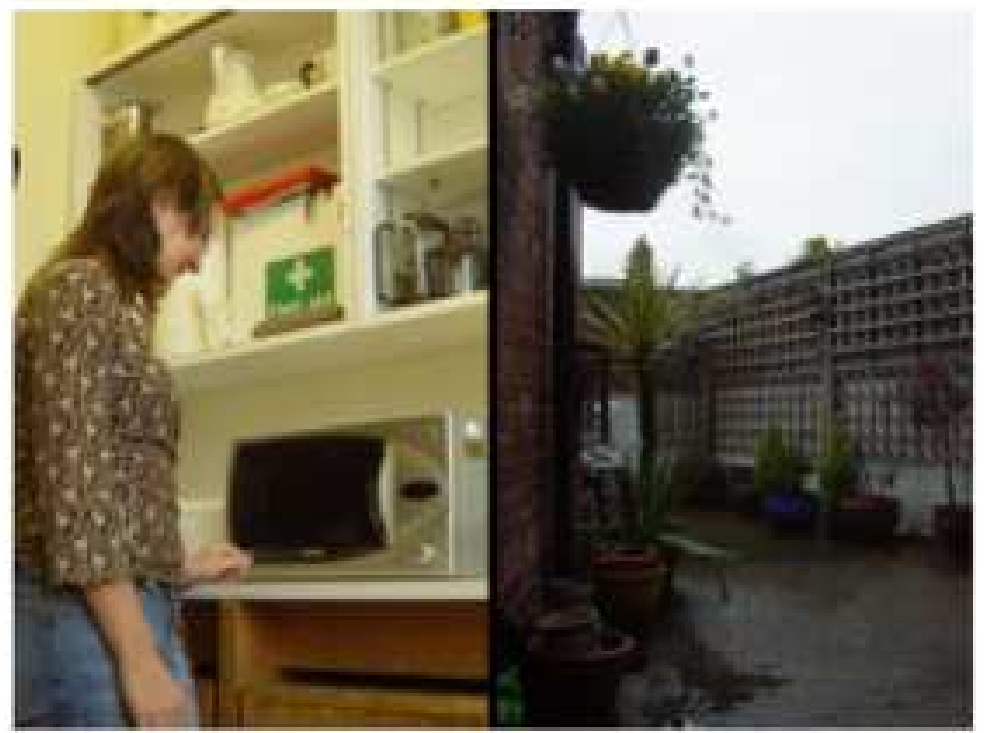

Fig 1a: Sample stimulus

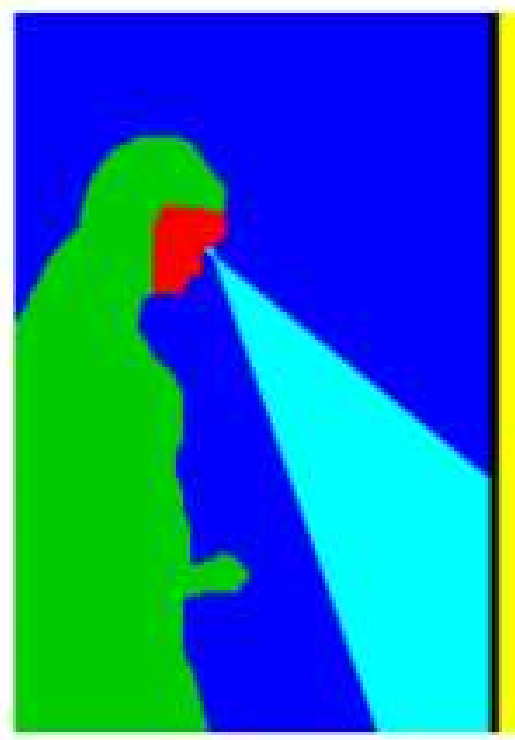

Fig 1c: Viewing cone domain

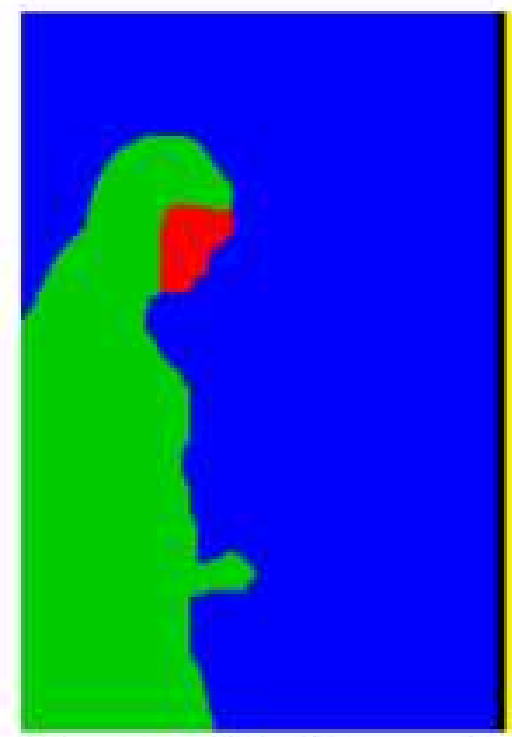

Fig 1b: Basic analysis domains

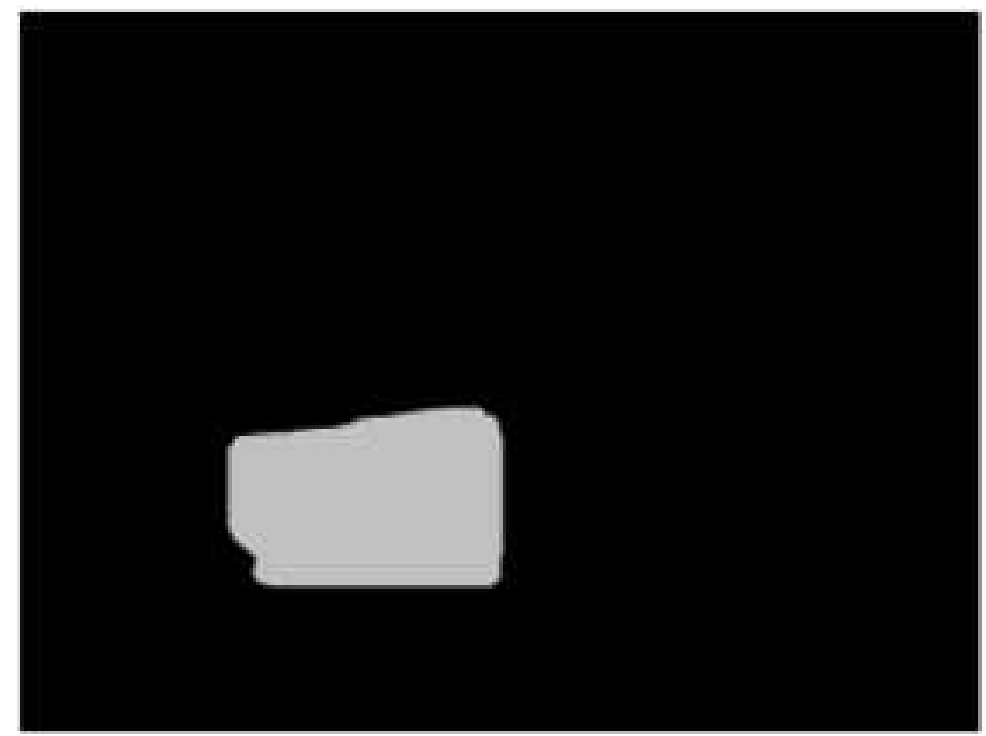

Fig 1d: Object domain 
Click here to download Figure: Fig 2 and Fig 3.doc

Figure 2:

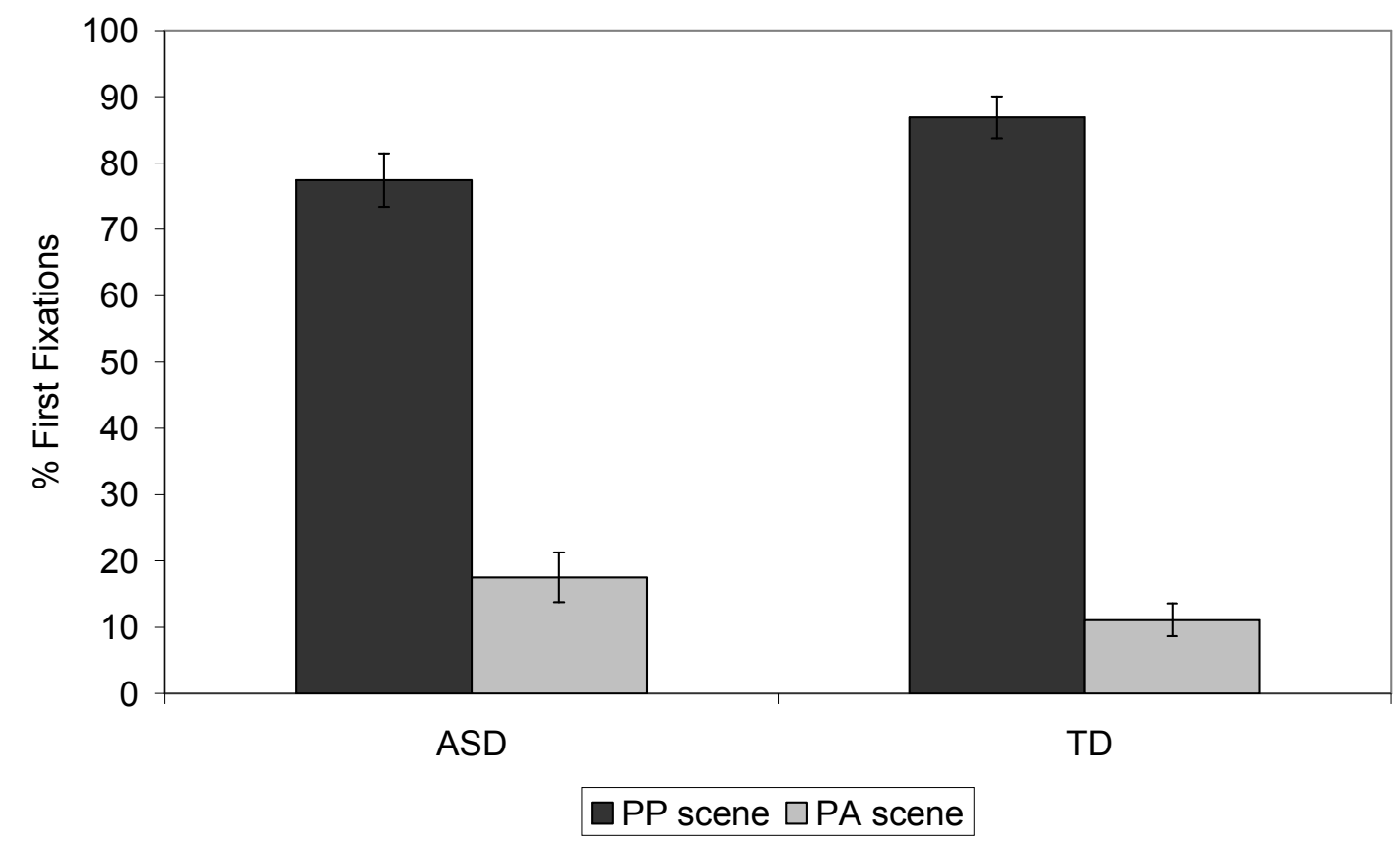


Figure 3:

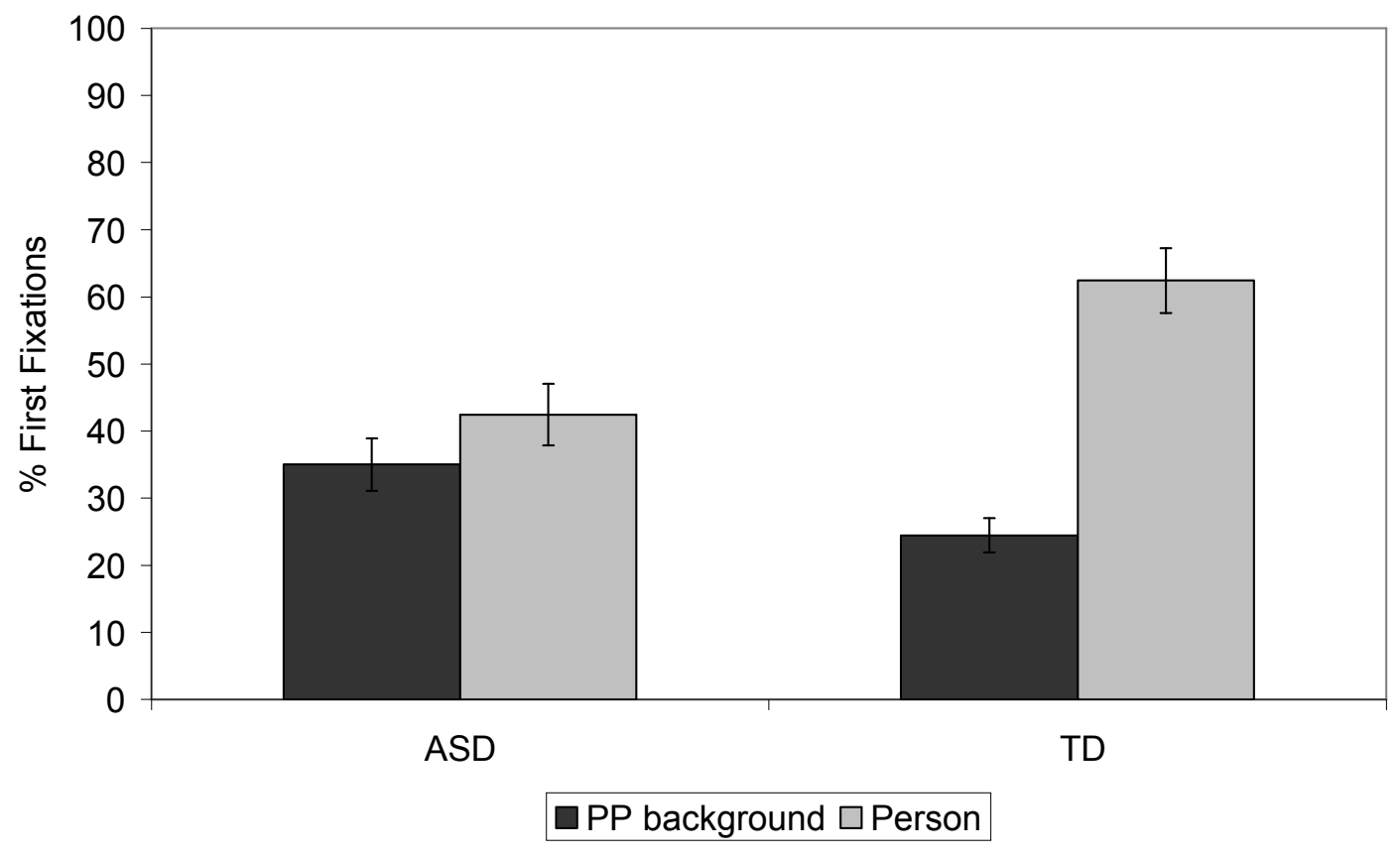


Click here to download high resolution image
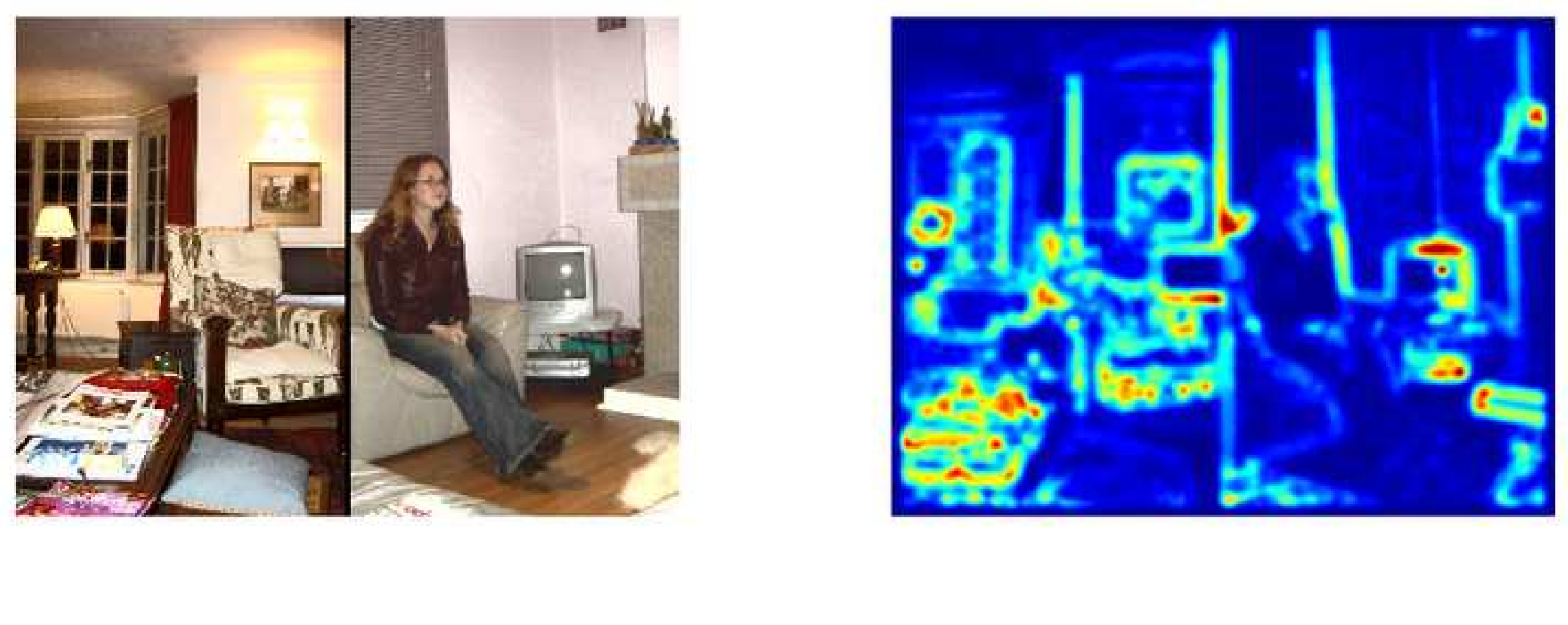


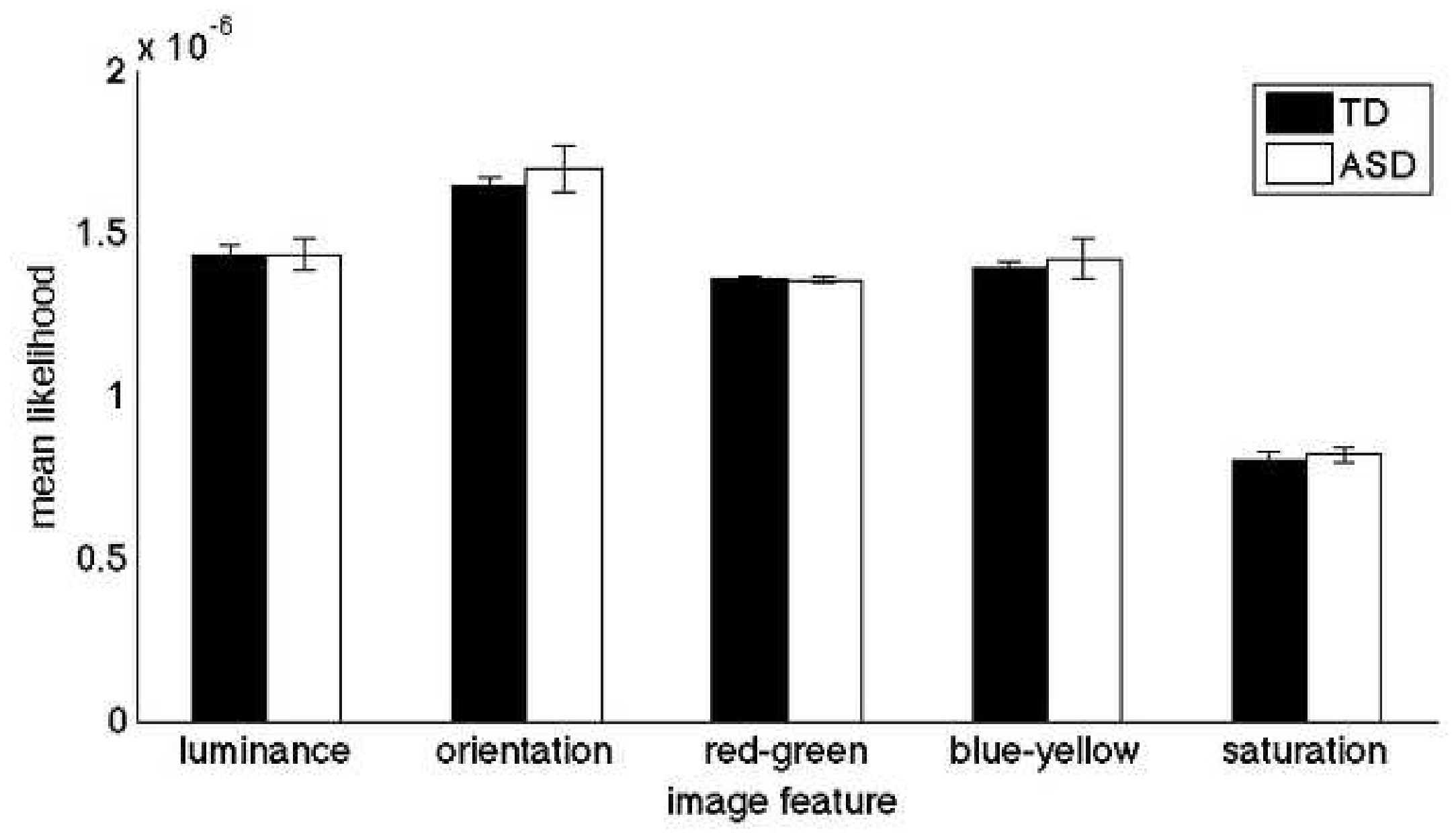

